\title{
miRNA activity inferred from single cell mRNA expression
}

\begin{abstract}
Morten Muhlig Nielsen ${ }^{1,2}$ \& Jakob Skou Pedersen ${ }^{1,2,3 \bowtie}$
High throughput single-cell RNA sequencing (scRNAseq) can provide mRNA expression profiles for thousands of cells. However, miRNAs cannot currently be studied at the same scale. By exploiting that miRNAs bind well-defined sequence motifs and typically down-regulate target genes, we show that motif enrichment analysis can be used to derive miRNA activity estimates from scRNAseq data. Motif enrichment analyses have traditionally been used to derive binding motifs for regulatory factors, such as miRNAs or transcription factors, that have an effect on gene expression. Here we reverse its use. By starting from the miRNA seed site, we derive a measure of activity for miRNAs in single cells. We first establish the approach on a comprehensive set of bulk TCGA cancer samples $(n=9679)$, with paired mRNA and miRNA expression profiles, where many miRNAs show a strong correlation with measured expression. By downsampling we show that the method can be used to estimate miRNA activity in sparse data comparable to scRNAseq experiments. We then analyze a human and a mouse scRNAseq data set, and show that for several miRNA candidates, including liver specific miR-122 and muscle specific miR-1 and miR-133a, we obtain activity measures supported by the literature. The methods are implemented and made available in the miReact software. Our results demonstrate that miRNA activities can be estimated at the single cell level. This allows insights into the dynamics of miRNA activity across a range of fields where scRNAseq is applied.
\end{abstract}

The introduction of single-cell RNA sequencing (scRNAseq) is having an immense impact on our understanding of spatial and temporal gene expression dynamics and is allowing studies of gene expression in rare cell types. As most scRNAseq techniques rely on primer extension from the polyadenylated tail of messenger RNAs (mRNAs), they primarily quantify protein-coding transcripts. Other classes of transcripts and in particular microRNAs (miRNAs), cannot be studied as readily or at the same scale. Though progress has been made on measuring miRNA expression in single cells, original fluorescence-based methods can only evaluate a few miRNAs per cell $^{1-3}$, while recent sequencing-based methods require extensive cell handling and thus have only been applied to few cells ${ }^{4-7}$. Single cell miRNA sequencing is thus still not standard practice and the study of functional roles of miRNAs thus lack behind that of mRNAs. Methods that can increase our understanding of miRNAs at the single cell level are likely to provide additional insight into their functional roles.

Here we explore the potential to evaluate miRNA activity levels based on mRNA expression profiles. We exploit that miRNAs typically destabilise their targets and that each miRNA can bind to the 3'UTRs of many mRNAs. The activity of a miRNA can thus be evaluated statistically by considering the relative expression levels of all target genes.

$\mathrm{We}^{8}$ and others ${ }^{9-13}$ have previously developed statistical methods that exploit this idea to study sequence motifs. These approaches have typically identified motifs that associate with expression levels based on case and control experiments, e.g. with overexpression of a molecule of interest, such as a miRNA ${ }^{9-13}$. We here reverse this setup and exploit that the binding motifs (target sites) of miRNAs are known. We can thereby evaluate miRNA activity scores based on expression profiles across data sets with many samples.

We first demonstrate that miRNA activity scores, inferred from bulk mRNA expression profiles, correlate with bulk miRNA expression levels across a set of nearly ten thousand pan-cancer samples from The Cancer Genome Atlas (TCGA) ${ }^{14}$. These observations further extend to a set of nearly eight thousand normal tissue samples from $\mathrm{GTEx}^{15}$. By downsampling, we reveal a potential for using this approach in sparse scRNAseq datasets. Focussing on miR-122, which is known to be highly tissue-specific ${ }^{16}$, we provide a proof of principle for the use of miRNA activity scores in both mouse and human scRNAseq datasets and confirm that miR-122 is primarily active in liver, and at the single cell level, primarily in hepatocytes. We further show examples of other miRNAs with tissue and cell type specific activity scores.

${ }^{1}$ Department of Molecular Medicine (MOMA), Aarhus University Hospital, Palle Juul-Jensens Boulevard 99, 8200 Aarhus N, Denmark. ²Department of Clinical Medicine, Aarhus University, Palle Juul-Jensens Boulevard 82, 8200 Aarhus N, Denmark. ${ }^{3}$ Bioinformatics Research Centre, C.F. Møllers Allé 8, Aarhus University, 8000 Aarhus C, Denmark. ${ }^{\square}$ email: jakob.skou@clin.au.dk 
As single cell miRNA sequencing remains challenging to perform at scale, the miReact approach offers an opportunity to study miRNA biology in large-scale single cell experiments, at no additional cost over scRNAseq. The power and scope to infer single cell miRNA activities from scRNAseq experiments will further benefit from a continued increase in read depth and cellular throughput.

\section{Results}

Inferring miRNA activity from bulk mRNA expression profiles. We first sought to establish whether miRNA acitivity could be inferred from mRNA expression profiles from bulk-tissue RNAseq. For this, we extracted 9679 mRNA expression profiles from TCGA cancer samples ${ }^{14}$, most of which had matched miRNA expression ( $n=9366$; Fig. 1a). The basic idea of the approach is to evaluate if genes with miRNA target sites (binding motifs) are downregulated compared to other genes. To make expression levels comparable between genes, the expression levels are traditionally transformed into fold-changes based on a control sample, as used by related motif-analysis methods ${ }^{8-10,13}$. As we lack control samples in our setting, we calculate fold-changes relative to the median gene expression level across all samples (Fig. 1b).

We thereafter evaluate the activity score for a comprehensive set of miRNAs ${ }^{17}(n=2047)$ for each sample. For each miRNA we annotate the $3^{\prime}$ UTR sequences with presence of binding sites (Fig. 1d). We exploit that miRNA binding is primarily defined by the $5^{\prime}$ seed site-a seven nucleotide long motif that has perfect complementarity to the miRNA targets ${ }^{18}$. miRNA binding models have proven efficient to evaluate activity of ectopically expressed miRNAs in cell line experiments in vitro ${ }^{8-10,12,13}$. For each sample, the 3'UTR sequences are ranked by their relative expression and the presence of motifs scored (Fig. 1c). Finally, miRNA activity is statistically evaluated as the association of 3'UTR motif enrichments with gene expression, using either of three previously developed measures $^{8,9}$ (Fig. 1e,f). For further details, see Methods and Supplemental note.

miRNA activity as a miRNA expression proxy. As a proof of concept, we first focussed on the activity scores for miR-122, which is an established liver specific miRNA. miR-122 has more activity in liver cancer samples than in samples with other tissues of origin (Fig. 1g). This also corresponds well with the miR122 expression levels observed across the same samples (Fig. 1h), which indeed are positively correlated with the activity scores both in the liver cohort $(\mathrm{R}=0.58, \mathrm{p}<2 \mathrm{e}-16$, Fig. $1 \mathrm{i})$ and across all cancer types $(\mathrm{R}=0.85$, $p<2 e-16$, Fig. 2a). This pattern is absent for the target of miR-122-3p, the opposite strand to the mature miRNA miR-122-5p, thus supporting that this approach can evaluate the activity of miRNAs in a strand specific manner (Supplemental Figure 1).

We next performed a more comprehensive evaluation of how different types of activity scores correlate with miRNA expression across the TCGA data set. More concretely, we compared four different ways of calculating motif enrichment scores. The first relies on a modified rank sum (mrs) statistic and the second on a brownian bridge (bb) statistic, both implemented in Regmex ${ }^{8}$. The third relies on the Sylamer method, which is based on a hyper geometric statistic ${ }^{9}$. Finally, the fourth method relies on rank sum statistics, but is based on miRNA targets defined in the TarBase database ${ }^{19}$ rather than motifs. The activity scores for all methods are the negative log of enrichment p-values times the direction of the enrichment, thus all four methods report signed enrichment, such that positive values (high miRNA activity) indicate enrichment of motifs in repressed genes. For miR-122, all three motif based approaches assign high scores to the liver cancer samples (Fig. 1g and Supplemental Figure 2) and significant correlations between expression and activity, with the mrs performing best (Fig. 2a). The method using TarBase targets did not show a similar shift for liver samples (Supplemental Figure 2). We then repeated this analysis across all miRNAs in our set and ranked them by their maximal correlation across all four ways of calculating activity scores. Among top-50, the mrs method most often showed the highest correlation (Fig. 2b). We thus used the mrs method for the remaining analysis. The full method is implemented and made available in the miReact tool.

Cases of tissue specific miRNA activity. Having established that miRNA activities can be inferred from gene expression of TCGA samples, using miR-122 as a case study, we looked further into some of the other miRNAs with high activity scores and strong miRNA expression correlation (Fig. 2b). We expanded the analysis to include the comprehensive set of healthy human tissue samples from the GTEx data set ${ }^{15}$, which again showed the expected liver-specific expression pattern for miR-122 (Supplemental Figure 3).

miR-9 and miR-124 are both top-ranked by miRNA expression correlation in TCGA (Fig. 2b) and both known to be expressed in brain tissue ${ }^{20,21}$. They primarily show activity in gliomas (LGG) and glioblastomas (GBM, Supplemental Figure 4). The activity in the glioma samples are significantly correlated with miRNA expression. The glioblastoma samples do not have miRNA expression measures in the TCGA data set, however, based on these results, we hypothesize that miR-9 is expressed. Similar expression patterns are observed In the healthy brain tissue from GTEx (Supplemental Figure 3).

We then looked at miR-1, a muscle specific miRNA ${ }^{22,23}$, which shows activity in GTEx skeletal muscle and heart tissues, as we would also expect (Supplemental Figure 3). Finally, we looked at miR-7, a miRNA that was shown to bind to the circular RNA circ7as, which regulates its activity by acting as a miRNA sink ${ }^{24,25}$. Here we saw a higher activity in the pituitary gland, hypothalamus, and adrenal gland, in line with a neuroendocrine role for miR-7 in human, as has been established in fish, mouse, and pig $^{26-29}$ (Supplemental Figure 3).

Downsampling suggests applicability at the single cell level. The above results suggest that this approach might be used as an indicator of miRNA activity in single cell expression (scRNAseq) experiments. However, of primary concern is the data sparsity of such experiments. To investigate the potential, we created down-sampled versions of the TCGA data set. We assigned a total number of reads for each sample, and sampled 
a

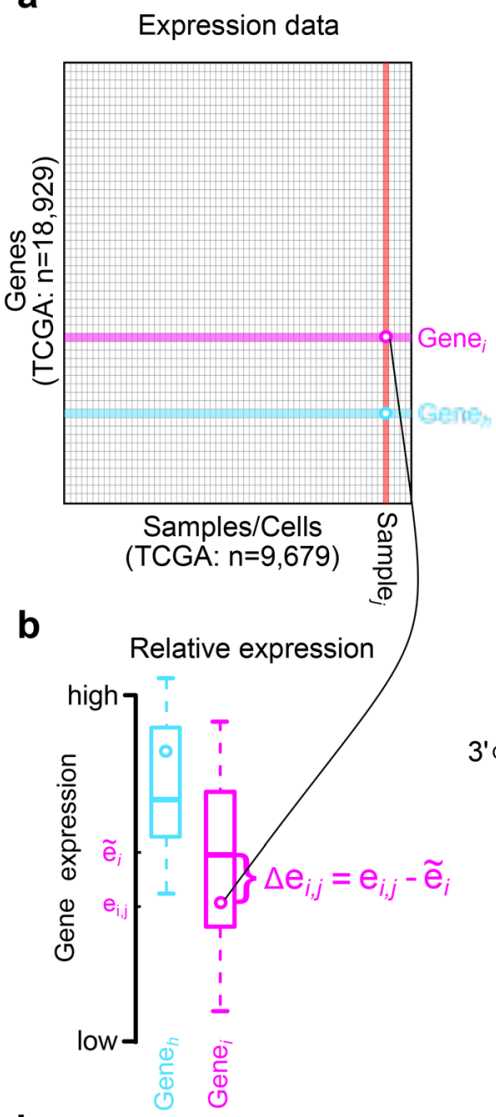

i

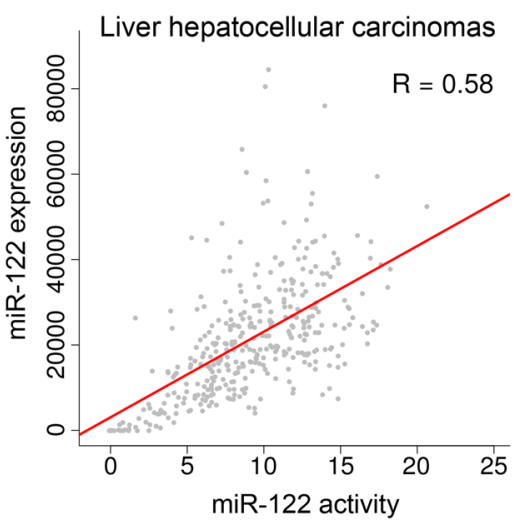

C

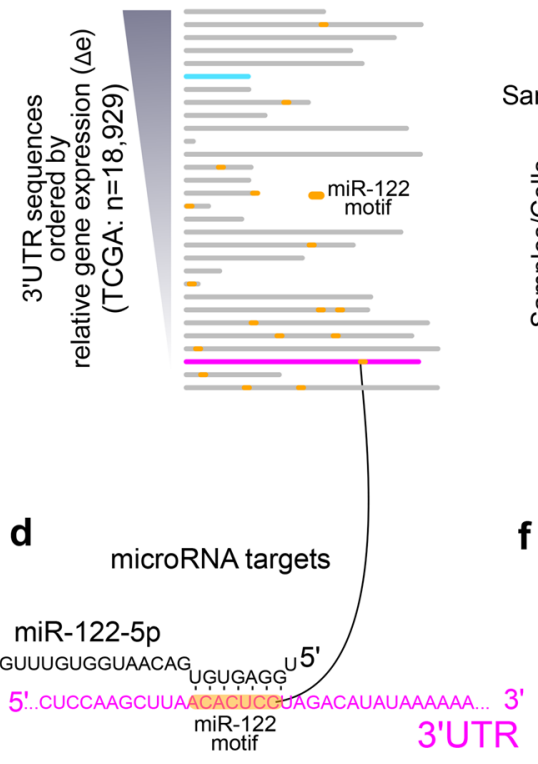

g

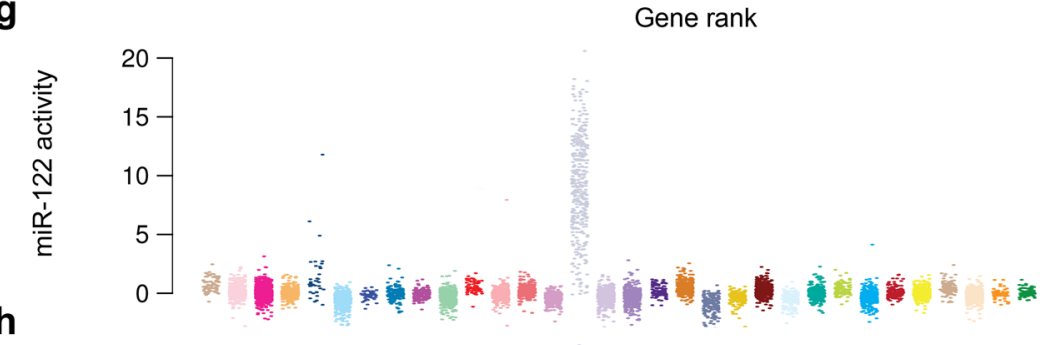

microRNA activity scores

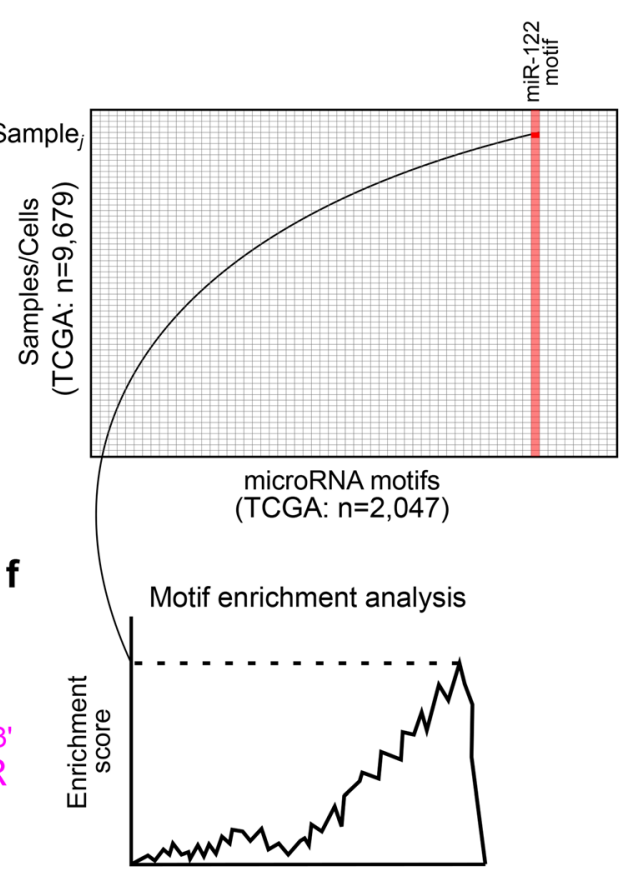

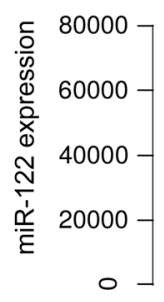

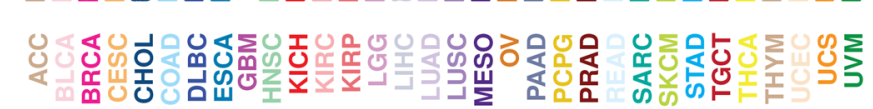

Figure 1. Method overview and illustration on miR-122 and TCGA data. Gene level expression data (a) is converted into sample/cell relative expression $(\Delta \mathrm{e})(\mathbf{b})$ by subtraction of gene medians (denoted by ) obtained across all samples/cells. For each sample/cell, 3'UTR sequences sorted by relative gene expression (c) are annotated with miRNA motif presence (d) and subjected to motif enrichment analysis evaluating motifs' association with expression (f). miRNA activity scores (e) are derived from the motif enrichment statistics to produce scores for each individual sample/cell for all miRNA binding motifs. $\mathbf{g}, \mathbf{h}$ Activity scores for miR122-5p (g) and miR-122 expression (h) in TCGA samples. TCGA abbreviations are in Supplemental Table 1. i, Correlations between miR-122-5 activity scores and miR-122 expression across liver hepatocellular carcinomas (LIHC).

reads proportional to each genes' expression value, thus simulating a situation of sparsity. We then ran miReact for different numbers of total reads spanning from 1000 to 10,000,000 reads per sample. scRNAseq experiments can provide meaningful cell type classification at library sizes above 10,000 reads $^{30,31}$ and expression level saturation occurs at around $1,000,000$ reads $^{32}$.

We observed that even at 1000 reads, we see positive, albeit small, correlations with expression for the top correlating miRNAs (Fig. 2c,d, Supplemental Figure 5). Increasing the reads cause correlations to rapidly increase towards the values of the full TCGA expression set. In particular, going from 10,000 to 50,000 reads has the largest effect on the correlations for many miRNAs. There is thus reason to attempt to apply the method on scRNAseq expression data to see if already known findings can be reproduced. 
a
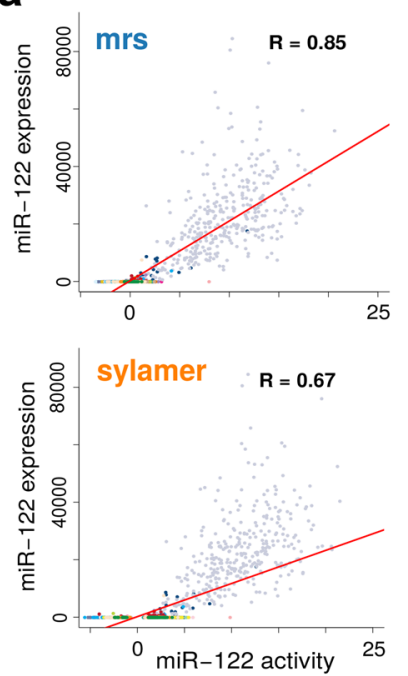
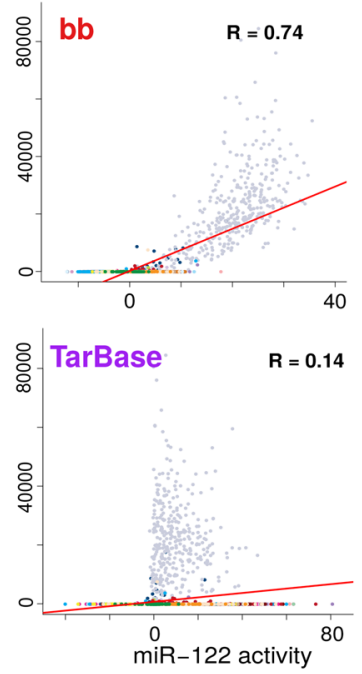

b

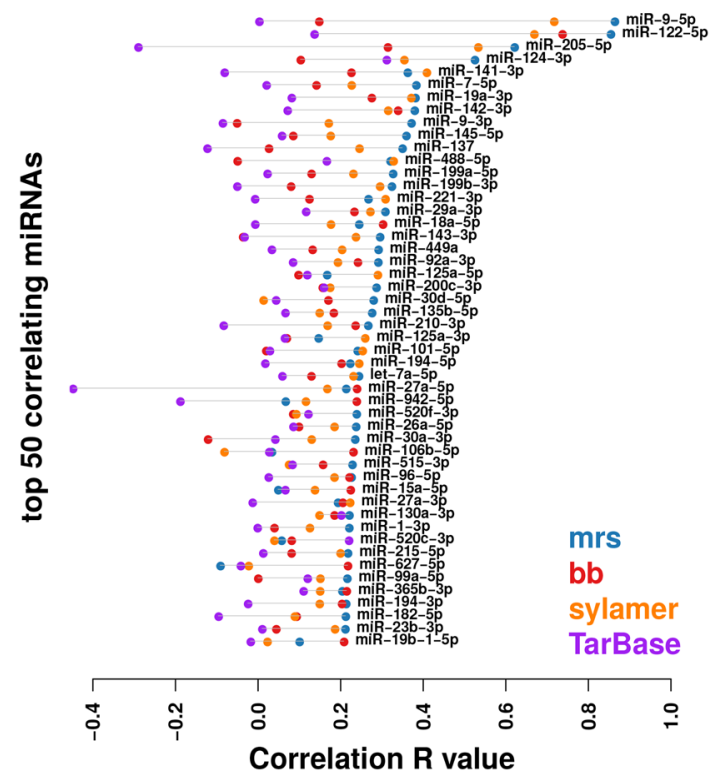

C
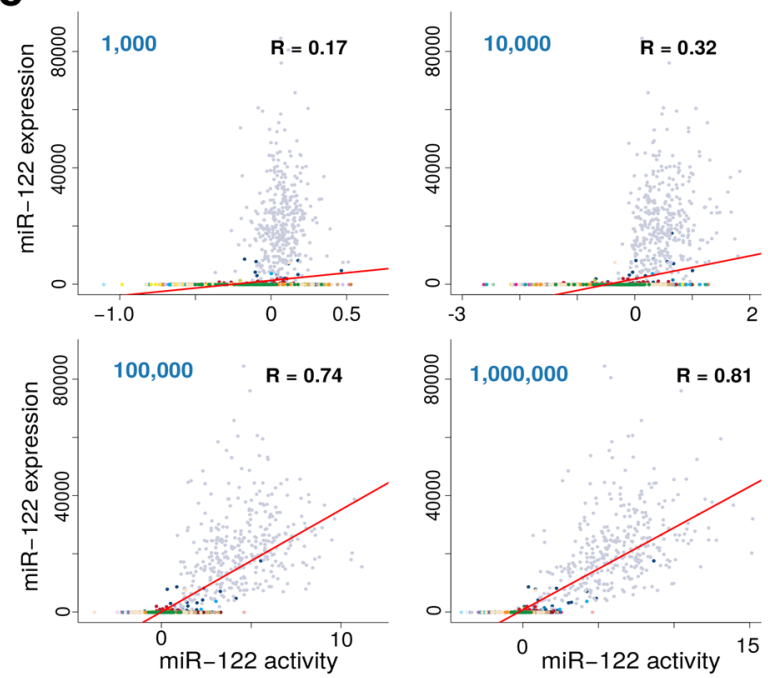

d

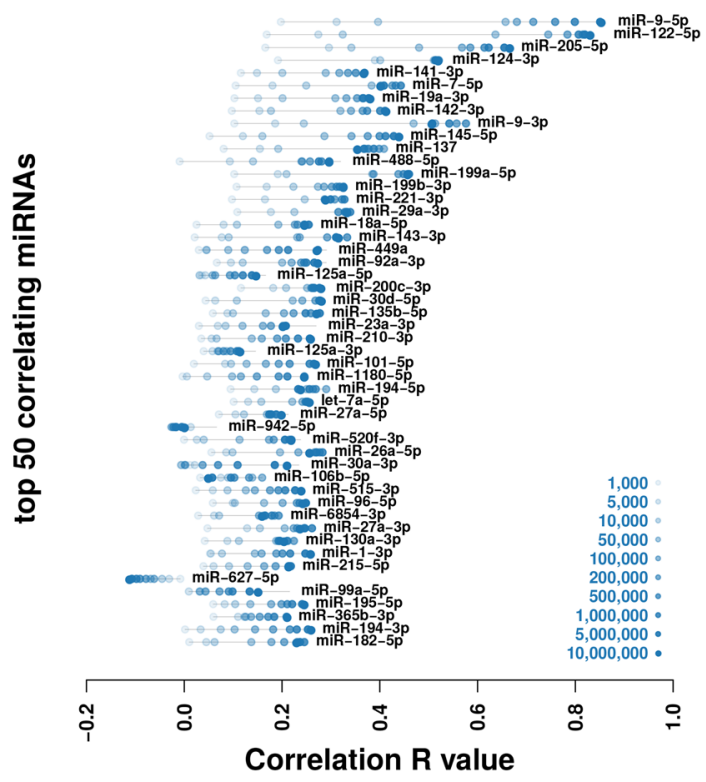

Figure 2. Method evaluation. a Correlation of miR-122 activity with expression for the four different motif enrichment methods across the TCGA data set $(n=9679)$. b Correlation values for the 50 miRNAs with highest correlation between activity and expression for any of the four activity score evaluation methods. $\mathrm{c}$ plots as in (a) based on the mrs method for downsampled TCGA data sets. d Correlation values for top 50 miRNAs as in (b) based on the mrs method for 10 different downsampled TCGA data sets with indicated number of total read counts per sample (shades of blue).

miR-122 activity in single cells. We first investigated miR-122 activity in two scRNAseq data sets: The 'Tabula Muris' of 20 mouse organs that contains 44,949 FACS sorted cells ${ }^{33}$, which has a high read count (median $>400,000$ reads/cell, see methods) and a human liver experiment with 10,372 cells ${ }^{34}$ and a much lower read count (median < 3000 reads/cell). For the mouse data set, as with TCGA and GTEx, we saw that miR-122 activity was higher in liver tissue, and thus at the single cell level could reproduce this observation (Fig. 3a-c).

Unlike the bulk liver samples, there were many mouse liver cells with low miR-122 activity. We further looked into this by sub-setting the liver cells based on the cell type annotations provided by the consortium ${ }^{33}$. Here we observed, as expected, that miR-122 activity mainly resides in hepatocytes, and correlates with expression of HNF4alpha, a transcription factor known to regulate miR-122 ${ }^{35,36}$ (Fig. 3d,e). Moreover, the expression of two known targets of miR-122, Racl and $\mathrm{RhoA}^{36}$, correlates negatively with the miRNA activity (Fig. 3f,g). To validate further, we looked at expressions of experimentally validated mouse miR-122 targets defined in the TarBase database. We observed that miR-122 targets generally had lower expression in cells with higher miR122 activity (Supplemental Figure 6). This thus supports that miRNA binding motif enrichment analysis may be useful for the study of miRNA activity at the single cell level. There were hepatocytes which appeared not to have any miR-122 activity. These cells had a low number of expressed genes $(<2000)$, indicating a limit in sensitivity when expression data is sparse (Supplemental Figure 7). 
a

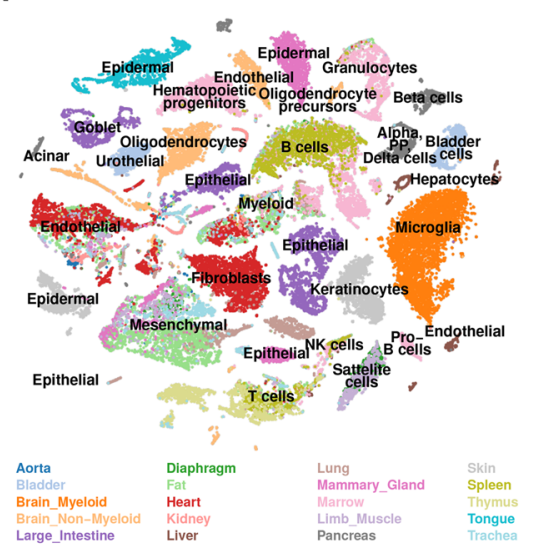

b

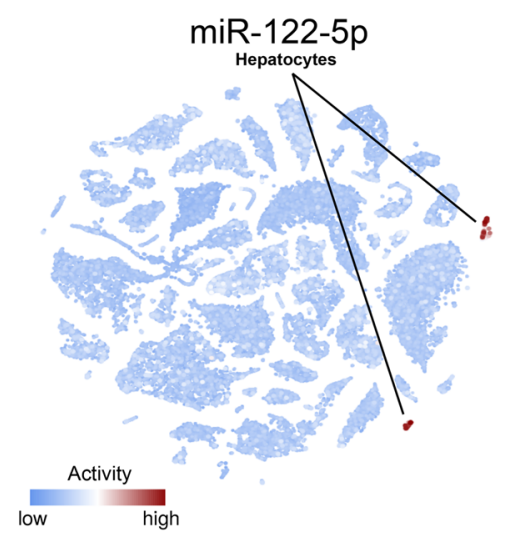

C

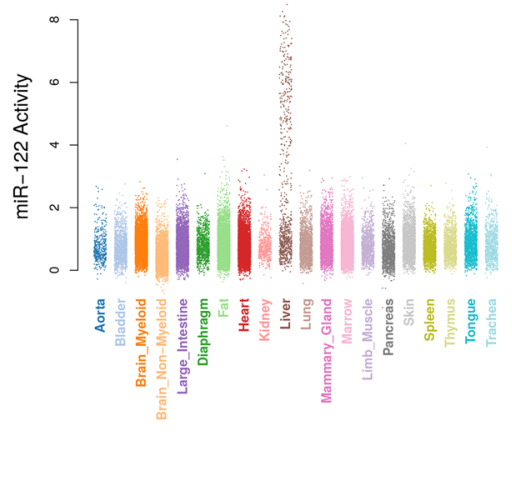

d

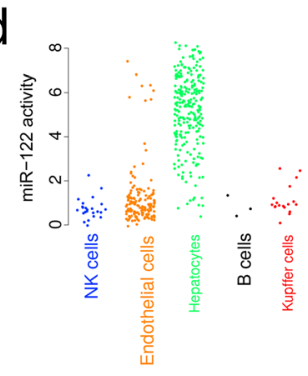

h

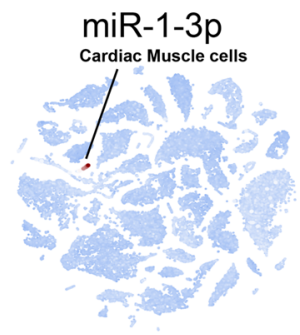

e

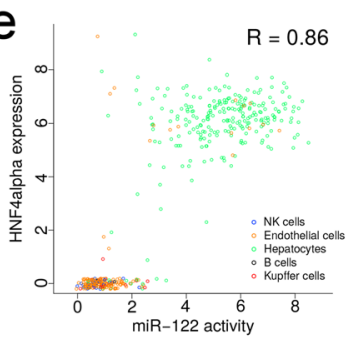

$f$

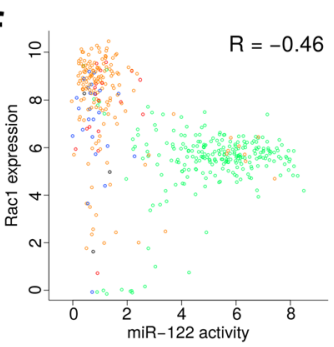

9

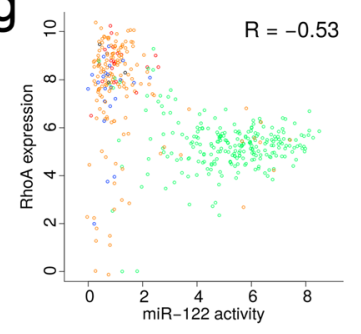

Figure 3. miRNA activity in single cells. a t-SNE plot showing clusters of cell types colored by tissue types for 42,192 cells of the Tabula Muris data set. Embeddings and cell annotations were obtained from the original work. b miR-122 activity overlaid the t-SNE embeddings as in (a) with indication of cell types for high activity cells. $\mathbf{c}$ miR-122 activity of individual cells separated into tissues. $\mathbf{d}$ miR-122 activity of individual liver cells separated into cell types. e,f,g Correlation between mir-122 activity and expression of the miR-122 transcription factor HNF4alpha (e), and miR-122 targets $\operatorname{Rac1}(\mathbf{f})$, and RhoA (g). h miRNA activities overlaid t-SNE embeddings as in (a) with indication of cell types for high activity cells. Cells with less than 2000 expressed genes were removed in $(\mathbf{d}-\mathbf{g})$.

In the human liver data set, despite a 100 fold lower median read count, we also saw an increased miR-122 activity in hepatocytes as well as in EPCAM + cells relative to other cell types, although here the signal was less pronounced (Supplemental Figure 8a-c). Again, there is a positive correlation with HNF4alpha expression as well as negative correlations with both target genes, but here as well, the effect was smaller than in the mouse data set (Supplemental Figure 8d-f). This is likely due to low read counts in some hepatocytes (Supplemental Figure 9).

Cell type specific miRNA activity. Discovery of cell type specific miRNA activity in single cell data sets opens for analysis of miRNAs in rare cell types that can be difficult to obtain with traditional sampling techniques. We thus looked for additional examples of miRNAs with high activity in a single or few cell types in the mouse data set. By comparing the miRNA activity distributions for each cell type, scoring for cell types with outlier behaviour, we identified several miRNAs with cell type specific activity. Muscle specific miRNAs miR-1 and miR-133 were active in cardiac muscle cells, but had low activity in other cell types of the heart including smooth muscle cells (Fig. 3, Supplemental Figure 10). miR-124 activity was specific to neuronal brain cells and miR-29a and miR-7 showed activity in pancreatic cells as well as neuronal cells. This generally fits with observations in the bulk data sets and the literature ${ }^{20,37}$, although we did not observe increased activity of miR-7 in pancreas samples from GTEx (Supplemental Figure 3). miR-7 has been reported to be active in pancreas in both mouse and human in a cell specific manner ${ }^{38,39}$. It is thus possible that a bulk experiment would conceal a signal from the target genes because of dilution by other cell types. 


\section{Discussion}

We have shown that motif enrichment analysis can be useful to estimate activity of miRNAs in high-throughput expression experiments, including scRNAseq. Measuring miRNA expression has often not been a priority in consortia, despite the importance of understanding these regulatory molecules. At the single cell level, current protocols lack the possibility of creating miRNA expression at a high-throughput scale. This method can thus be a useful tool for the study of miRNAs under conditions where expression measures are not easily obtainable.

Yet, far from all miRNAs show a good correlation between calculated activity and expression. A number of reasons may explain this. The activity measure is based on reduced expression of mRNAs with miRNA targets. However, besides leading to mRNA degradation, miRNAs are known to also exert their effect by inhibiting translation without reduction in mRNA expression ${ }^{40}$. Thus this effect may be absent from the activity signal. Other factors than seed site complementarity, e.g. local mRNA structure, can also be important for binding ${ }^{18}$. The 3'UTR model is based on the longest sequence among potentially multiple isoforms, some of which could be tissue specific, and thus the model for some genes in some samples or cells could be wrong. Finally, the model for the expected gene expression, the median across the expression set, does not accurately reflect gene expression in the absence of the miRNA, but rather is a surrogate. It has the advantage over merely using sample gene expression, that genes turned off in most samples will not be ranked extreme in general.

We note that for a miRNA to show good activity to expression correlation in a dataset, it needs to hold some variation in expression of a miRNA. This is not necessarily the case in the TCGA dataset. An example is miR-1, which is active in muscle tissue. Since there are no muscle-derived samples in the TCGA cancer set, we saw only a modest correlation between activity and expression. For the GTEx and mouse single cell data set, however, we saw a highly specific signal in muscle and heart tissue, clearly indicating that activity reflects expression of miR-1, although not demonstrable in the TCGA dataset. Overall, we observed that miRNAs with good correlation between miReact activity and expression had high miRNA expression variation across the TCGA data set (standard deviation $>100$, supplemental Figure 11). Low variance in the TCGA data set may thus conceal how the method performs for many miRNAs, as demonstrated for miR-1 above. For $75 \%$ of the miRNAs, expression standard deviations were below 100 in the TCGA data set.

However, among the $25 \%$ miRNAs with high expression variance across samples, most correlations were low, indicating that there is room for improvement in the method for these miRNAs. It is possible that the 7-mer motif used as a binding model may be insufficient or wrong for some of the miRNAs. Using elaborate and realistic binding models, such as accounting for G/U base pairing between miRNA and target or allowing for multiple motifs to define binding sequences, may be paths to improved performance for the method.

We conducted an analysis using experimentally verified targets from TarBase, and included the option of using these as input to the miReact procedure. We found, however, that this performed consistently worse than the methods relying on motifs (Fig. 2b). Running the method with TarBase targets reduces the binding information to a logical measure, and thus power can be lost when evaluating the enrichment statistics. This may be a reason for the worse performance. We note that in the case of miR-122, we observed a low but positive correlation (0.14, Fig. 2a) in the human TCGA set, yet for the mouse single cell data set, we were able to validate the finding of lowered expression of miR-122 targets upon higher miR-122 activity (Supplemental Figure 6). In line with this, we observed that more motif-containing UTRs were found among mouse TarBase targets (45\%) than in human (17\%). Also, nearly four fold more targets are reported for mouse (6471) than for human (1737). It could thus indicate that for miR-122, the TarBase target set may lack some targets in human, which may explain a poor performance.

Inferring miRNA activity may provide additional insight over measuring expression. Although we saw a good correlation between miR-7 activity and expression, miR-7 sequestration into a complex with the circular RNA circ7as ${ }^{24,25}$ may decouple activity from expression, and activity may thus add to the analysis of the function of this complex. In cases where miRNA expression is measured at the premature level, activity calculation could provide insight into the usage of the mature strands. For instance, for miR-122 it is clear that $5 p$ is the active strand.

We showed by downsampling that good correlations between expression and activity were obtainable with read counts well below current standards for scRNAseq libraries. We further demonstrated the use of the method on two scRNAseq datasets in mouse and human and with relative high and low read coverage. This demonstrates that the method may be used to infer miRNA activity at the single cell level, and as such should be a useful hypothesis generating tool for studying miRNAs.

Importantly, this type of analysis applied to large datasets could easily be extended beyond miRNAs and 3'UTR sequences. A close analogy lies in transcription factors binding to promoter sequences and RNA binding proteins binding to 3'UTRs. This type of approach may thus facilitate studies of regulatory mechanisms in single cells.

\section{Online Methods}

Single cell expression data. For both single cell data sets, we used the following procedure to create inputs for miRNA activity calculations: Cells with less than 1000 mapped reads across all genes were removed. Counts were divided by the total count in each cell and multiplied by the max total count across all cells. Counts were added a pseudocount of one and logged (base 2).

3'UTR sequences. We used 3'UTR sequences as provided by GENCODE v. 19. If genes had multiple transcripts with different $3^{\prime} \mathrm{UTR}$ lengths, we used the longest form. We removed genes with $3^{\prime} \mathrm{UTR}$ lengths less than 20 bases and longer than 10,000 bases. 
Fold change value calculations. We used logged expression values as described above, and calculated a fold change value for each gene in each sample or cell by subtracting an expected expression value for that gene. The expected expression value was calculated as the median expression value across all samples or cells in an analysis run, e.g. for TCGA this would be the median expression value across all 9679 samples.

miRNA binding model. For all miRNAs, we used the 7-mer sequence complementary to the miRNA seed site as the motif defining miRNA binding sites. We used miRNA mature sequences as defined in miRbase v.2017 and used bases from position 2 to 8 from the 5 'end as the seed site definition. For the TarBase approach, we used target interactions as defined in TarBase v. $8^{19}$.

Probability of motifs in sequences. For each 7-mer miRNA binding model, we used Regmex to calculate probabilities for observing the 7-mer motif in sequences similar (equal length and base composition) to the 3'UTR sequence of each gene. Such probabilities were harvested for all 7-mers and 3'UTRs.

miRNA activity calculation. For each sample or cell, we ordered genes by their fold change values. We next calculated miRNA activity from the probability output of three different motif enrichment tools, Regmex ${ }^{8}$ in brownian bridge (bb) as well as modified rank sum (mrs) mode (see Supplemental Note) and Sylamer' We used the ordered 3'UTR sequences as input for Sylamer.

Similarly for the Regmex methods, we used the ordered 3'UTR sequences and pre-calculated motif probabilities in the sequences as input, and calculated miRNA activity scores as $-\log 10(\mathrm{p})^{\star} \operatorname{sign}($ statistics).

We provide a tool, miReact, available at https://github.com/muhligs/miReact that implements the method using the modified rank sum (mrs) mode for use on large expression data sets. See Supplemental note for a tutorial of running miReact on the Tabula Muris data set.

\section{Data availability}

The 'Tabula Muris' data set is available as count data at (https://doi.org/10.6084/m9.figshare.5829687.v7).

The human liver data set is available in the Gene Expression Omnibus (GEO) with the accession code GSE124395. See supplemental note for further processing of the data.

\section{Code availability}

The miReact software is written in R and available under the MIT license at https:/github.com/muhligs/miReact.

Received: 17 September 2020; Accepted: 8 April 2021

Published online: 28 April 2021

\section{References}

1. Porichis, F. et al. High-throughput detection of miRNAs and gene-specific mRNA at the single-cell level by flow cytometry. Nat. Commun. 5, 5641 (2014).

2. Guo, S. et al. Ultrahigh-throughput droplet microfluidic device for single-cell miRNA detection with isothermal amplification. Lab Chip 18, 1914-1920 (2018).

3. Wu, M., Piccini, M., Koh, C.-Y., Lam, K. S. \& Singh, A. K. Single cell microRNA analysis using microfluidic flow cytometry. PLoS ONE 8, e55044 (2013).

4. Alberti, C. et al. Cell-type specific sequencing of microRNAs from complex animal tissues. Nat. Methods 15, 283-289 (2018).

5. Xiao, Z. et al. Holo-Seq: single-cell sequencing of holo-transcriptome. Genome Biol. 19, 163 (2018).

6. Wang, N. et al. Single-cell microRNA-mRNA co-sequencing reveals non-genetic heterogeneity and mechanisms of microRNA regulation. Nat. Commun. 10, 95 (2019).

7. Faridani, O. R. et al. Single-cell sequencing of the small-RNA transcriptome. Nat. Biotechnol. 34, 1264-1266 (2016).

8. Nielsen, M. M., Tataru, P., Madsen, T., Hobolth, A. \& Pedersen, J. S. Regmex: A statistical tool for exploring motifs in ranked sequence lists from genomics experiments. Algorithms Mol. Biol. 13, 17 (2018).

9. van Dongen, S., Abreu-Goodger, C. \& Enright, A. J. Detecting microRNA binding and siRNA off-target effects from expression data. Nat. Methods 5, 1023-1025 (2008).

10. Rasmussen, S. H., Jacobsen, A. \& Krogh, A. cWords-Systematic microRNA regulatory motif discovery from mRNA expression data. Silence 4, 2 (2013).

11. Eden, E., Lipson, D., Yogev, S. \& Yakhini, Z. Discovering motifs in ranked lists of DNA sequences. PLoS Comput. Biol. 3, e39 (2007).

12. Leibovich, L., Paz, I., Yakhini, Z. \& Mandel-Gutfreund, Y. DRIMust: A web server for discovering rank imbalanced motifs using suffix trees. Nucleic Acids Res. 41, W174-W179 (2013).

13. Steinfeld, I., Navon, R., Ach, R. \& Yakhini, Z. miRNA target enrichment analysis reveals directly active miRNAs in health and disease. Nucleic Acids Res. 41, e45 (2013).

14. The Cancer Genome Atlas Research Network et al. The Cancer Genome Atlas Pan-Cancer analysis project. Nat. Genet. 45, 11131120 (2013).

15. Carithers, L. J. et al. A novel approach to high-quality postmortem tissue procurement: The GTEx Project. Biopreserv. Biobank. 13, 311-319 (2015).

16. Lagos-Quintana, M. et al. Identification of tissue-specific microRNAs from mouse. Curr. Biol. 12, 735-739 (2002).

17. Kozomara, A. \& Griffiths-Jones, S. miRBase: annotating high confidence miRNAs using deep sequencing data. Nucleic Acids Res. 42, D68-D73 (2014).

18. McGeary, S. E. et al. The biochemical basis of microRNA targeting efficacy. Science 366, eaav1741 (2019).

19. Karagkouni, D. et al. DIANA-TarBase v8: a decade-long collection of experimentally supported miRNA-gene interactions. Nucleic Acids Res. 46, D239-D245 (2017).

20. Smirnova, L. et al. Regulation of miRNA expression during neural cell specification. Eur. J. Neurosci. 21, 1469-1477 (2005).

21. Deo, M., Yu, J.-Y., Chung, K.-H., Tippens, M. \& Turner, D. L. Detection of mammalian microRNA expression by in situ hybridization with RNA oligonucleotides. Dev. Dyn. 235, 2538-2548 (2006).

22. Lim, L. P. et al. Microarray analysis shows that some microRNAs downregulate large numbers of target mRNAs. Nature 433, 769-773 (2005). 
23. Zhao, Y., Samal, E. \& Srivastava, D. Serum response factor regulates a muscle-specific microRNA that targets Hand2 during cardiogenesis. Nature 436, 214-220 (2005).

24. Hansen, T. B. et al. Natural RNA circles function as efficient microRNA sponges. Nature 495, 384-388 (2013).

25. Memczak, S. et al. Circular RNAs are a large class of animal RNAs with regulatory potency. Nature 495, 333-338 (2013).

26. Tessmar-Raible, K. et al. Conserved sensory-neurosecretory cell types in annelid and fish forebrain: insights into hypothalamus evolution. Cell 129, 1389-1400 (2007).

27. He, J. et al. MiR-7 mediates the zearalenone signaling pathway regulating FSH synthesis and secretion by targeting FOS in female pigs. Endocrinology 159, 2993-3006 (2018)

28. Li, X. et al. miR-7 mediates the signaling pathway of NE affecting FSH and LH synthesis in pig pituitary. J. Endocrinol. 244, 459-471 (2020).

29. Ahmed, K. et al. Loss of microRNA-7a2 induces hypogonadotropic hypogonadism and infertility. J. Clin. Invest. 127, 1061-1074 (2017).

30. Jaitin, D. A. et al. Massively parallel single-cell RNA-seq for marker-free decomposition of tissues into cell types. Science $\mathbf{3 4 3}$, 776-779 (2014).

31. Pollen, A. A. et al. Low-coverage single-cell mRNA sequencing reveals cellular heterogeneity and activated signaling pathways in developing cerebral cortex. Nat. Biotechnol. 32, 1053-1058 (2014).

32. Ziegenhain, C. et al. Comparative Analysis of Single-Cell RNA Sequencing Methods. Mol. Cell 65, 631-643.e4 (2017).

33. Tabula Muris Consortium et al. Single-cell transcriptomics of 20 mouse organs creates a Tabula Muris. Nature 562, 367-372 (2018).

34. Aizarani, N. et al. A human liver cell atlas reveals heterogeneity and epithelial progenitors. Nature 572, 199-204 (2019).

35. Li, Z.-Y. et al. Positive regulation of hepatic miR-122 expression by HNF4a. J. Hepatol. 55, 602-611 (2011).

36. Wang, S.-C. et al. MicroRNA-122 triggers mesenchymal-epithelial transition and suppresses hepatocellular carcinoma cell motility and invasion by targeting RhoA. PLoS ONE 9, e101330 (2014).

37. Oliveira-Carvalho, V., da Silva, M. M. F., Guimarães, G. V., Bacal, F. \& Bocchi, E. A. MicroRNAs: new players in heart failure. Mol. Biol. Rep. 40, 2663-2670 (2013).

38. Correa-Medina, M. et al. MicroRNA miR-7 is preferentially expressed in endocrine cells of the developing and adult human pancreas. Gene Expr. Patterns 9, 193-199 (2009).

39. Nieto, M. et al. Antisense miR-7 impairs insulin expression in developing pancreas and in cultured pancreatic buds. Cell Transpl. 21, 1761-1774 (2012).

40. Eulalio, A., Huntzinger, E. \& Izaurralde, E. Getting to the root of miRNA-mediated gene silencing. Cell 132, 9-14 (2008).

\section{Acknowledgements}

We thank Lasse Maretty Sørensen and Christian Kroun Damgaard for valuable comments to the manuscript. J.S.P. and MMN were funded by the Independent Research Fund Denmark | Medical Sciences (DFF-7016-00379) and The Novo Nordic Foundation (NNF18OC0053222).

\section{Author contributions}

M.M.N. and J.S.P. Planned the project. M.M.N. wrote the code and performed analysis. M.M.N. and J.S.P. wrote the manuscript.

\section{Competing interests}

The authors declare no competing interests.

\section{Additional information}

Supplementary Information The online version contains supplementary material available at https://doi.org/ 10.1038/s41598-021-88480-5.

Correspondence and requests for materials should be addressed to J.S.P.

Reprints and permissions information is available at www.nature.com/reprints.

Publisher's note Springer Nature remains neutral with regard to jurisdictional claims in published maps and institutional affiliations.

(c) (i) Open Access This article is licensed under a Creative Commons Attribution 4.0 International License, which permits use, sharing, adaptation, distribution and reproduction in any medium or format, as long as you give appropriate credit to the original author(s) and the source, provide a link to the Creative Commons licence, and indicate if changes were made. The images or other third party material in this article are included in the article's Creative Commons licence, unless indicated otherwise in a credit line to the material. If material is not included in the article's Creative Commons licence and your intended use is not permitted by statutory regulation or exceeds the permitted use, you will need to obtain permission directly from the copyright holder. To view a copy of this licence, visit http://creativecommons.org/licenses/by/4.0/.

(c) The Author(s) 2021, corrected publication 2022 